\title{
A PHASE-BASED IMAGE REGISTRATION ALGORITHM FOR DENTAL RADIOGRAPH IDENTIFICATION
}

\author{
Akira Nikaido $^{1}$, Koichi Ito ${ }^{1}$, Takafumi Aoki ${ }^{1}$, Eiko Kosuge ${ }^{2}$ and Ryota Kawamata ${ }^{2}$ \\ ${ }^{1}$ Graduate School of Information Sciences, Tohoku University, \\ 6-6-05, Aramaki Aza Aoba, Aoba-ku, Sendai-shi, 980-8579 Japan \\ E-mail: \{nikaido, ito\}@aoki.ecei.tohoku.ac.jp \\ 2 Department of Oral and Maxillofacial Radiology, Kanagawa Dental College, \\ 82, Inaoka, Yokosuka-shi, 238-8580 Japan
}

\begin{abstract}
Dental radiographs have been used for the accurate assessment and treatment of dental diseases. For an accurate diagnosis, the complete geometric registration between radiographs is required. The perspective projection between two radiographs may be observed, even if they are taken from the same oral regions of the subject. This paper presents an efficient dental radiograph registration algorithm using Phase-Only Correlation (POC) function. The use of phase components in 2D (two-dimensional) discrete Fourier transforms of dental radiograph images makes possible to achieve highly robust image registration and recognition. Experimental evaluation using a dental radiograph database indicates that the proposed algorithm exhibits efficient recognition performance even for distorted radiographs.
\end{abstract}

Index Terms - biomedical image processing, image registration, medical diagnosis, dental radiograph, phase-only correlation

\section{INTRODUCTION}

Dental radiographs have been used for detecting small changes of internal bone structures, monitoring disease progression, planning and guiding dental treatment, etc. For these purposes, radiographs acquired from subjects over short or long periods of time have to be compared after image registration [1]. The accuracy of dental radiograph registration is important for an accurate diagnosis.

Intraoral X-ray examinations are performed as follows: (i) place a small imaging plate behind the teeth to be irradiated, (ii) bring the $\mathrm{X}$-ray tube close to the face, and (iii) irradiate the imaging plate (Fig. 1 (a)). In order to obtain the ideal radiograph, the imaging plate is placed parallel to the teeth and perpendicular to the plane of the $\mathrm{X}$ ray beam. Since the positions of the X-ray tube and the imaging plate are set manually by a radiologist, the geometric transformation between two radiographs taken from the same oral regions is given by the perspective projection as shown in the cases (i)-(iii) of Fig. 1. An image registration technique with projective distortion correction is required for the accurate diagnosis.

Addressing this problem, some algorithms of dental radiograph registration with projective distortion correction have been proposed $[2,3]$. These algorithms determine the landmark points on the registered image, obtain the corresponding points on the input image, and estimate the parameters of the projective transformation. Although a fully automatic registration algorithm is desired, most of the algorithms employ a manual selection of the corresponding points. Also,

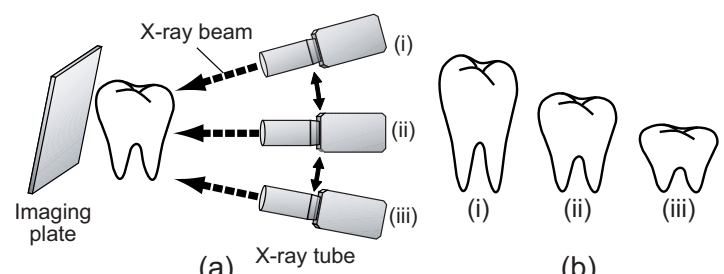

(a)

(b)

Fig. 1. Perspective projection between dental radiographs: (a) an intraoral X-ray examination, and (b) radiographs taken from the same tooth at different positions.

the registration performance of these algorithms is evaluated using only a small radiograph database.

In this paper, we propose an efficient algorithm for dental radiograph image registration using Phase-Only Correlation (POC) technique - an image matching technique using the phase components in 2D Discrete Fourier Transforms (DFTs) of given images. This technique has been successfully applied to sub-pixel image registration tasks for computer vision applications $[4,5,6]$ and similarity evaluation tasks for biometric authentication applications [7, 8, 9]. The proposed algorithm employs (i) high-accuracy image matching technique using POC function for rotation and displacement alignment and (ii) sub-pixel correspondence search technique using POC function for parameter estimation of projective transformation. The use of POC technique makes possible to achieve fully automatic and high-accuracy image registration even for distorted radiographs. Experimental evaluation using a set of dental radiographs taken before and after dental treatment demonstrates efficient registration performance of the proposed algorithm.

\section{PHASE-ONLY CORRELATION}

\subsection{Phase-only correlation function}

We introduce the principle of a Phase-Only Correlation (POC) function (which is sometimes called the "phase-correlation function") $[4,5,6]$.

Consider two $N_{1} \times N_{2}$ images, $f\left(n_{1}, n_{2}\right)$ and $g\left(n_{1}, n_{2}\right)$, where we assume that the index ranges are $n_{1}=-M_{1}, \cdots, M_{1}\left(M_{1}>0\right)$ and $n_{2}=-M_{2}, \cdots, M_{2}\left(M_{2}>0\right)$ for mathematical simplicity, and hence $N_{1}=2 M_{1}+1$ and $N_{2}=2 M_{2}+1$. Let $F\left(k_{1}, k_{2}\right)$ and $G\left(k_{1}, k_{2}\right)$ denote the 2D DFTs of the two images. $F\left(k_{1}, k_{2}\right)$ is 
given by

$$
\begin{aligned}
F\left(k_{1}, k_{2}\right) & =\sum_{n_{1}, n_{2}} f\left(n_{1}, n_{2}\right) W_{N_{1}}^{k_{1} n_{1}} W_{N_{2}}^{k_{2} n_{2}} \\
& =A_{F}\left(k_{1}, k_{2}\right) e^{j \theta_{F}\left(k_{1}, k_{2}\right)}
\end{aligned}
$$

where $k_{1}=-M_{1}, \cdots, M_{1}, k_{2}=-M_{2}, \cdots, M_{2}, W_{N_{1}}=e^{-j \frac{2 \pi}{N_{1}}}$, $W_{N_{2}}=e^{-j \frac{2 \pi}{N_{2}}}$, and $\sum_{n_{1}, n_{2}}$ denotes $\sum_{n_{1}=-M_{1}}^{M_{1}} \sum_{n_{2}=-M_{2}}^{M_{2}} . A_{F}\left(k_{1}, k_{2}\right)$ is amplitude and $\theta_{F}\left(k_{1}, k_{2}\right)$ is phase. $G\left(k_{1}, k_{2}\right)$ is defined in the same way. The cross-phase spectrum $R_{F G}\left(k_{1}, k_{2}\right)$ is given by

$$
R_{F G}\left(k_{1}, k_{2}\right)=\frac{F\left(k_{1}, k_{2}\right) \overline{G\left(k_{1}, k_{2}\right)}}{\left|F\left(k_{1}, k_{2}\right) \overline{G\left(k_{1}, k_{2}\right)}\right|}=e^{j \theta\left(k_{1}, k_{2}\right)},
$$

where $\overline{G\left(k_{1}, k_{2}\right)}$ is the complex conjugate of $G\left(k_{1}, k_{2}\right)$ and $\theta\left(k_{1}, k_{2}\right)$ denotes the phase difference $\theta_{F}\left(k_{1}, k_{2}\right)-\theta_{G}\left(k_{1}, k_{2}\right)$. The POC function $r_{f g}\left(n_{1}, n_{2}\right)$ is the 2D Inverse DFT (2D IDFT) of $R_{F G}\left(k_{1}, k_{2}\right)$ and is given by

$$
r_{f g}\left(n_{1}, n_{2}\right)=\frac{1}{N_{1} N_{2}} \sum_{k_{1}, k_{2}} R_{F G}\left(k_{1}, k_{2}\right) W_{N_{1}}^{-k_{1} n_{1}} W_{N_{2}}^{-k_{2} n_{2}},
$$

where $\sum_{k_{1}, k_{2}}$ denotes $\sum_{k_{1}=-M_{1}}^{M_{1}} \sum_{k_{2}=-M_{2}}^{M_{2}}$. When two images are similar, their POC function gives a distinct sharp peak. When two images are not similar, the peak drops significantly. The height of the peak gives a good similarity measure for image matching, and the location of the peak shows the translational displacement between the images.

\subsection{Phase-based image matching}

Listed below are key features of the phase-based image matching using POC function, which are used in this paper.

- Translational displacement estimation

We have proposed a high-accuracy translational displacement estimation method, which employs (i) an analytical function fitting technique to estimate the sub-pixel position of the correlation peak, (ii) a windowing technique to eliminate the effect of periodicity in 2D DFT, and (iii) a spectrum weighting technique to reduce the effect of aliasing and noise [5]. Our experimental observation shows that POC-based matching can estimate displacement between two images with 0.01-pixel accuracy when image size is about $100 \times 100$ pixels.

- Sub-pixel correspondence search

We have developed an efficient method of sub-pixel correspondence matching, which employs (i) a coarse-to-fine strategy using image pyramids for robust correspondence search and (ii) a subpixel window alignment technique for finding a pair of corresponding points with sub-pixel displacement accuracy [10]. Experimental evaluation shows that the displacement between corresponding points with 0.05 -pixel accuracy when using $32 \times 32$-pixel matching window.

- Rotation angle estimation

We have extended the POC-based image matching to the registration for images including translation, rotation and scaling simultaneously [5]. In this paper, we focus on the rotation alignment using the POC-based image matching. We employ the polar mapping of the amplitude spectrum to transform the image rotation into image translation. The rotation angle is estimated by detecting the corresponding translational displacements by the above technique. Experimental evaluation shows that the rotation angle between two images with 0.05 -degree accuracy when image size is $128 \times 128$ pixels.

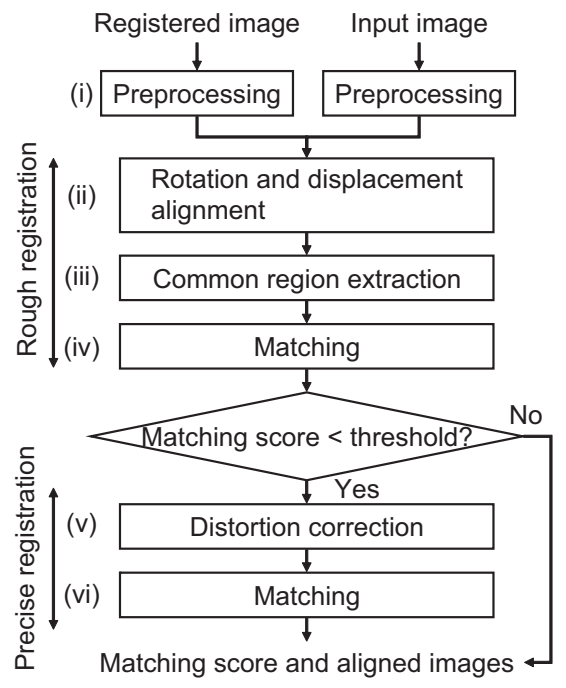

Fig. 2. Flow diagram of the proposed algorithm.

- Similarity evaluation

We have developed a similarity evaluation technique using BandLimited Phase-Only Correlation (BLPOC) function [7]. The idea to improve the similarity evaluation performance is to eliminate meaningless high frequency components in the calculation of cross-phase spectrum $R_{F G}$ depending on the inherent frequency components of images. Our experimental observation shows that the BLPOC function provides the higher correlation peak and better discrimination capability than that of the original POC function.

\section{DENTAL RADIOGRAPH REGISTRATION ALGORITHM}

In this section, we present a dental radiograph registration algorithm using the POC function. The proposed algorithm consists of the six steps: (i) preprocessing, (ii) rotation and displacement alignment, (iii) common region extraction, (iv) matching, (v) distortion correction and (vi) matching as shown in Fig. 2.

\section{(i) Preprocessing}

First step is the enhancement of radiograph image to allow accurate radiograph image processing, since these images are often blurred due to substantial noise, poor lighting, etc. In our proposed algorithm, we employ the contrast enhancement techniques using local area contrast enhancement [11] and morphological filters [12]. Figure 3 (b) shows the enhanced images, $f_{e}\left(n_{1}, n_{2}\right)$ and $g_{e}\left(n_{1}, n_{2}\right)$, of the registered image $f\left(n_{1}, n_{2}\right)$ and the input image $g\left(n_{1}, n_{2}\right)$, respectively.

\section{(ii) Rotation and displacement alignment}

We need to normalize rotation and displacement between the enhanced images $f_{e}\left(n_{1}, n_{2}\right)$ and $g_{e}\left(n_{1}, n_{2}\right)$ in order to perform the high-accuracy dental radiograph registration. We estimate the rotation angle $\theta$ by using POC technique. Using $\theta$, we obtain a rotationnormalized image $g_{e \theta}\left(n_{1}, n_{2}\right)$. Then, we align the translational displacement between $f_{e}\left(n_{1}, n_{2}\right)$ and $g_{e \theta}\left(n_{1}, n_{2}\right)$ using the peak location of the BLPOC function. Thus, we have normalized versions of the registered image and the input image as shown in Fig. 3 (c), which are denoted by $f^{\prime}\left(n_{1}, n_{2}\right)$ and $g^{\prime}\left(n_{1}, n_{2}\right)$.

(iii) Common region extraction

Next step is to extract the overlapped region (intersection) of the two images $f^{\prime}\left(n_{1}, n_{2}\right)$ and $g^{\prime}\left(n_{1}, n_{2}\right)$. This process improves the 


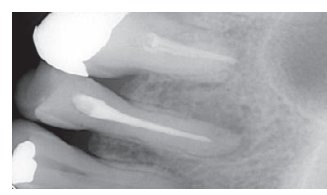

$f\left(n_{1}, n_{2}\right)$

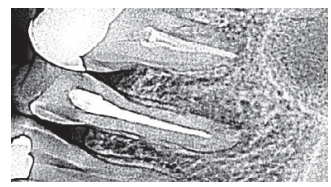

$f_{e}\left(n_{1}, n_{2}\right)$

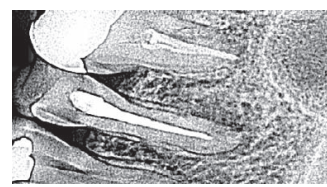

$f^{\prime}\left(n_{1}, n_{2}\right)$

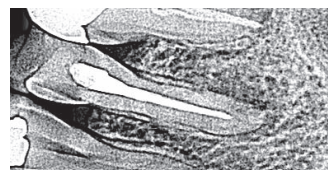

f" $\left(n_{1}, n_{2}\right)$

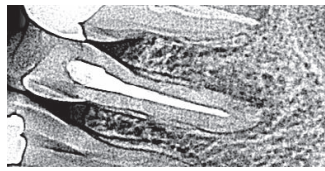

$f^{\prime \prime \prime}\left(n_{1}, n_{2}\right)$

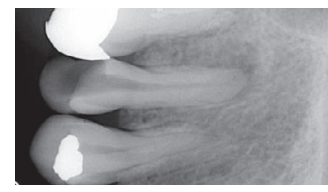

$g\left(n_{1}, n_{2}\right)$

(a)

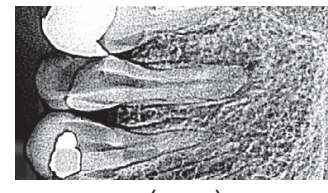

(b)

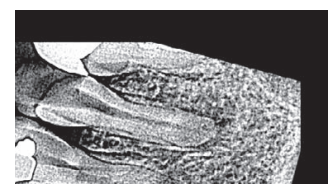

$g^{\prime}\left(n_{1}, n_{2}\right)$

(c)

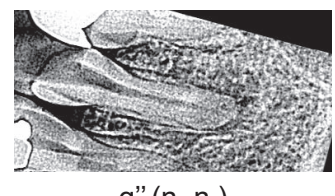

g" $\left(\mathrm{n}_{1}, \mathrm{n}_{2}\right)$

(d)

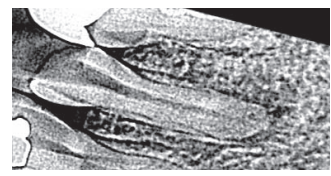

g'" $\left(n_{1}, n_{2}\right)$

(e)

Fig. 3. Example of dental radiograph registration: (a) the registered image and the input image, (b) the enhanced images, (c) the normalized images, (d) the extracted common regions and (e) images after distortion correction.
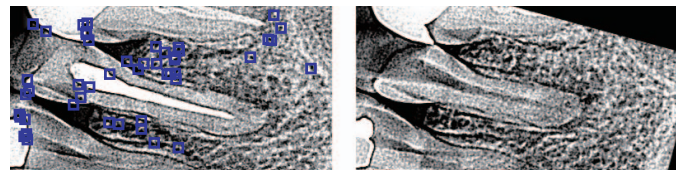

(a)

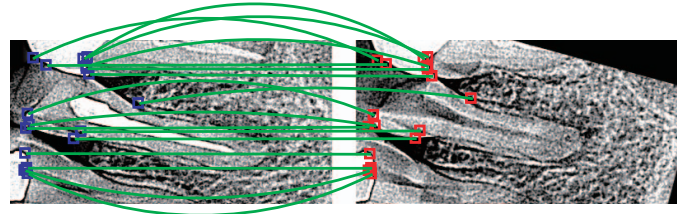

(b)

Fig. 4. Example of distortion correction: (a) common regions and extracted feature points on the registered image and (b) corresponding points between images.
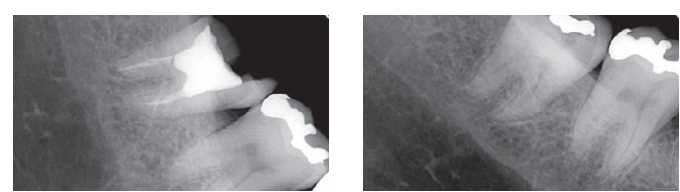

(a)
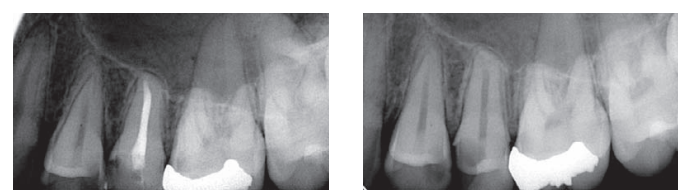

(b)

Fig. 5. Examples of dental radiographs in our database: the left-hand images are taken after dental treatment and the right-hand images are taken before dental treatment.

accuracy of dental radiograph matching, since the non-overlapped areas of the two images become the uncorrelated noise components in the BLPOC function. In order to detect the effective areas in $f^{\prime}\left(n_{1}, n_{2}\right)$ and $g^{\prime}\left(n_{1}, n_{2}\right)$, we examine the $n_{1}$-axis projection and the $n_{2}$-axis projection of pixel values. Only the common effective image areas, $f^{\prime \prime}\left(n_{1}, n_{2}\right)$ and $g^{\prime \prime}\left(n_{1}, n_{2}\right)$, with the same size are extracted for the succeeding image matching step (Fig. 3 (d)).

(iv) Matching

We calculate the BLPOC function between the two extracted images $f^{\prime \prime}\left(n_{1}, n_{2}\right)$ and $g^{\prime \prime}\left(n_{1}, n_{2}\right)$, and evaluate the matching score. The matching score is the highest peak value of the BLPOC function. (v) Distortion correction

If the matching score is less than the threshold, the projective distortion may be occurred between two images. We have to correct the projective distortion between two images. We first obtain the feature points in $f^{\prime \prime}\left(n_{1}, n_{2}\right)$ using the Harris corner detector [13] as shown in Fig. 4 (a). Next, we find the points in $g^{\prime \prime}\left(n_{1}, n_{2}\right)$ which correspond to the feature points in $f^{\prime \prime}\left(n_{1}, n_{2}\right)$ using the sub-pixel correspondence search using POC technique as shown in Fig. 4 (b). If the number of corresponding pairs is more than 4 , the parameters of the projective transformation can be estimated using the leastsquares method. Using these parameters, we obtain a distortioncorrected image $g^{\prime \prime \prime}\left(n_{1}, n_{2}\right)$. Thus, we obtain distortion-corrected images $f^{\prime \prime \prime}\left(n_{1}, n_{2}\right)$ and $g^{\prime \prime \prime}\left(n_{1}, n_{2}\right)$ as shown in Fig. 3 (e). (vi) Matching

To evaluate the matching score, we calculate the BLPOC function between two images $f^{\prime \prime \prime}\left(n_{1}, n_{2}\right)$ and $g^{\prime \prime \prime}\left(n_{1}, n_{2}\right)$. The matching score is the highest peak value of the BLPOC function.

\section{EXPERIMENTS}

This section describes a set of experiments using the dental radiograph database for evaluating registration performance of the proposed algorithm. In this experiment, we use dental radiographs taken before and after dental treatment. Our database consists of $120 \mathrm{im}-$ ages $(256 \times 460$ pixels $)$ with 60 subjects and 2 different images of each dental radiograph. Figure 5 shows some examples of the dental radiographs in this database.

We compare two algorithms: the proposed registration algorithm with distortion correction (A) and without distortion correction (B). The registration performance of the proposed algorithm is evaluated by identifying the subjects in order to perform the quantitative evaluation. In this experiment, 60 subjects after dental treatment 

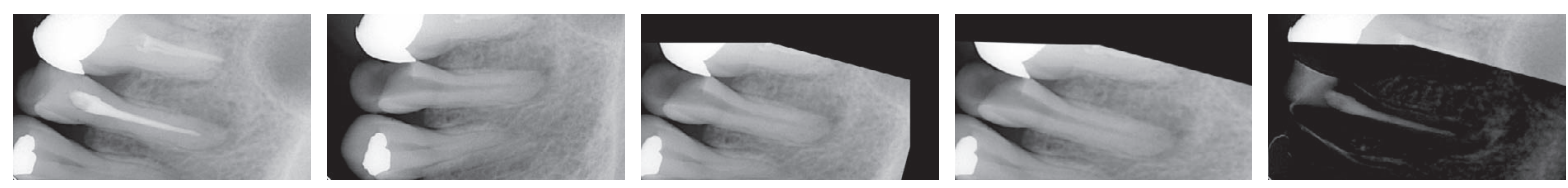

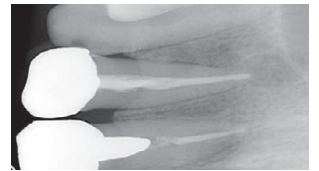

(a)

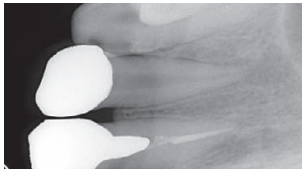

(b)

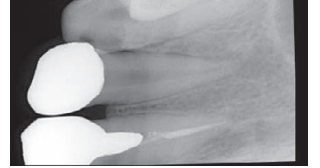

(c)

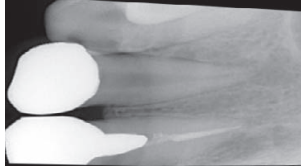

(d)

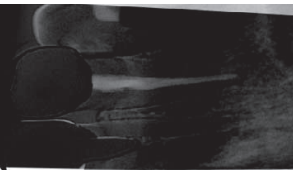

(e)

Fig. 7. Examples of registration results: (a) registered image $f\left(n_{1}, n_{2}\right)$, (b) input image $g\left(n_{1}, n_{2}\right)$, (c) rotation- and displacement-aligned image $g^{\prime}\left(n_{1}, n_{2}\right)$, (d) distortion-corrected image $g^{\prime \prime \prime}\left(n_{1}, n_{2}\right)$ and (e) subtraction image between $f\left(n_{1}, n_{2}\right)$ and $g^{\prime \prime \prime}\left(n_{1}, n_{2}\right)$.

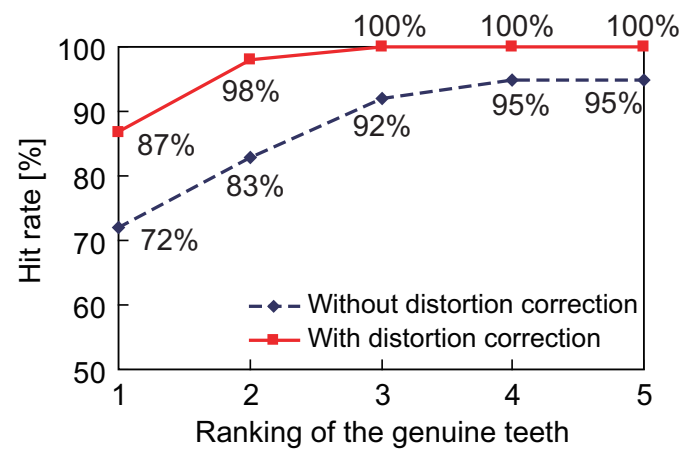

Fig. 6. Cumulative match curve of the proposed algorithm.

are matched to the 60 subjects before dental treatment. Figure 6 shows the cumulative match curve of the proposed algorithm, which is used for evaluating the performance of the identification system. Using the top-1 radiograph, the recognition accuracy of (A) is $87 \%$ $(=52 / 60)$ while that of $(B)$ is $72 \%(=43 / 60)$. As for the algorithm (A), the recognition accuracy reaches $100 \%$ when the top-3 radiographs are used. On the other hand, the algorithm (B) can not achieve $100 \%$ recognition accuracy even if the top-5 radiographs are used. Figure 7 shows examples of registration results and the subtraction image. As is observed in this experimental result, the proposed algorithm is useful for matching low-quality and distorted dental radiographs.

\section{CONCLUSION}

This paper proposed a dental radiograph registration algorithm using phase-only correlation technique. Experimental evaluation demonstrates efficient performance of our proposed algorithm for distorted dental radiographs. For our future work, we will develop a dental identification system using phase-only correlation technique and evaluate the performance of the developed system using a large-scale database of antemortem and postmortem dental radiographs.

\section{REFERENCES}

[1] D. L. G. Hill, P. G. Batchelor, M. Holden, and D. J. Hawkes, "Medical image registration," Phys. Med. Biol., vol. 46, pp. R1-R45, 2001.
[2] T. M. Lehmann, K. Gröndahl, H.-G. Gröndahl, W. Schmitt, and K. Spitzer, "Observer-independent registration of perspective projection prior to substraction of in vivo radiographs," Dentomaxillofacial Radiology, vol. 27, pp. 140-150, 1998.

[3] T. M. Lehmann, H.-G. Gröndahl, and D. K. Benn, "Computerbased registration for digital subtraction in dental radiology," Dentomaxillofacial Radiology, vol. 29, pp. 323-346, 2000.

[4] C. D. Kuglin and D. C. Hines, "The phase correlation image alignment method," Proc. Int. Conf. Cybernetics and Society, pp. 163-165, 1975.

[5] K. Takita, T. Aoki, Y. Sasaki, T. Higuchi, and K. Kobayashi, "High-accuracy subpixel image registration based on phaseonly correlation," IEICE Trans. Fundamentals, vol. E86-A, no. 8, pp. 1925-1934, Aug. 2003.

[6] K. Takita, M. A. Muquit, T. Aoki, and T. Higuchi, "A sub-pixel correspondence search technique for computer vision applications," IEICE Trans. Fundamentals, vol. E87-A, no. 8, pp. 1913-1923, Aug. 2004

[7] K. Ito, H. Nakajima, K. Kobayashi, T. Aoki, and T. Higuchi, "A fingerprint matching algorithm using phase-only correlation," IEICE Trans. Fundamentals, vol. E87-A, no. 3, pp. 682-691, Mar. 2004.

[8] K. Miyazawa, K. Ito, , T. Aoki, K. Kobayashi, and H. Nakajima, "A phase-based iris recognition algorithm," Lecture Notes in Computer Science (ICB2006), vol. 3832, pp. 356-365, Dec. 2005.

[9] K. Ito, T. Aoki, H. Nakajima, K. Kobayashi, and T. Higuchi, "A palmprint recognition algorithm using phase-based image matching," Proc. the 2006 IEEE Int. Conf. Image Processing, Oct. 2006.

[10] M. A. Muquit, T. Shibahara, and T. Aoki, "A high-accuracy passive $3 \mathrm{~d}$ measurement system using phase-based image matching," IEICE Trans. Fundamentals, vol. E89-A, no. 3, pp. 686-697, Mar. 2006.

[11] G. X. Ritter and J. N. Wilson, Handbook of Computer Vision Algorithms in Image Algebra, CRC Press, 1996.

[12] P. Soille, Morphological Image Analysis, Springer, 1999.

[13] C. Harris and M. Stephens, "A combined corner and edge detector," Proc. The Fourth Alvey Vision Conference, pp. 147151,1988 . 